\title{
Pontes conceituais entre matemática e redução de risco de desastres socioambientais - o conceito de bacia hidrográfica estocástica
}

\begin{abstract}
Resumo
A bacia hidrográfica é a unidade espacial básica em estudos hidrológicos. A modelagem deste objeto geográfico é de fundamental interesse para redução de risco de desastres socioambientais. Neste artigo são apresentados resultados da delimitação de bacias hidrográficas em uma perspectiva diferente da tradicional, proporcionando recursos de análise inovadores à modelagem de cenários de risco hidrológico. Trata-se da exploração do comportamento probabilístico da direção de escoamento superficial e da análise do efeito que uma pequena alteração local pode ter na estrutura geral das bacias.
\end{abstract}

Palavras-chave: Desastres Socioambientais. Bacia Hidrográfica. Matemática Aplicada. Processos Estocásticos. Mapas de Probabilidades.

\section{Leonardo Bacelar Lima Santos}

Bacharel em Física pela

Universidade Federal da Bahia -

UFBA. Pesquisador Adjunto do

Centro Nacional de

Monitoramento e Alertas de

Desastres Naturais - Cemaden.

Brasil

santoslbl@gmail.com

\section{Patrick Vasconcellos}

Graduando em Geografia na Universidade de Taubaté -

UNITAU. Bolsista de Iniciação Científica pelo Instituto Nacional de Pesquisas Espaciais - INPE.

Brasil

patrick.vasconcellos1@gmail.com

\section{Tiago Nogueira Sá Miranda}

Tecnólogo em Análise e Desenvolvimento de Sistemas pela Faculdade de Tecnologia -

FATEC. Bolsista de Iniciação Científica pelo Instituto Nacional de Pesquisas Espaciais - INPE. Brasil

tiago.n.miranda@gmail.com

\section{Solon Venâncio de Carvalho}

Bacharel em Engenharia

Eletrônica pelo Instituto

Tecnológico de Aeronáutica -

ITA. Pesquisador Senior do Instituto Nacional de Pesquisas

Espaciais - INPE

Brasil

soloncarvalho@gmail.com 


\title{
Conceptual bridges between mathematic and disaster risk reduction - the concept of Stochastic Watershed
}

\begin{abstract}
The drainage basin is the basic spatial unit for hydrological studies. The modeling of this geographic object is of main interest ondisasters risk reduction. In this paper we present the results of watershed delimitation in a different perspective from the traditional, providing innovative analysis to model hydrological risk scenarios. It was analysed the probabilistic behavior of the local drain directions and its effect on the general structure of the watershed.
\end{abstract}

Keywords: Natural Disasters. Watershed. Applied Mathematics. Stochastic Processes. Probability Maps.

\section{Para citar este artigo:}

SANTOS, Leonardo Bacelar Lima; VASCONCELLOS, Patrick; MIRANDA, Tiago Nogueira Sá; CARVALHO, Solon Venâncio de. Pontes conceituais entre matemática e redução de risco de desastres socioambientais - o conceito de bacia hidrográfica estocástica. Revista PerCursos, Florianópolis, v. 18, n.36, p. 125 - 136, jan./abr. 2017.

\section{DOI: $10.5965 / 1984724618362017125$}

http://dx.doi.org/10.5965/1984724618362017125 


\section{Introdução}

Dentre os diversos significados da expressão "inovar" está um referente à modificação/atualização de formas tradicionais de pensar. A inovação de um conceito de uma área do conhecimento pode emergir de um olhar vindo de outras áreas. A Matemática, pelo seu formalismo todo particular, e o forte caráter crítico das Ciências Sociais, podem, juntas, proporcionar uma valiosa fonte de inovações a diversas áreas, como, por exemplo, a hidrologia.

O Brasil é um país privilegiado quanto aos seus recursos hídricos. A região Amazônica, por exemplo, detém a maior bacia de água doce do mundo - esta ocupa uma área aproximada de $6.110 .000 \mathrm{~km}^{2}$, com contribuição hídrica de $73,6 \%$ em relação à disponibilidade total do país (AGÊNCIA NACIONAL DE ÁGUAS, 2017).

Vale destacar também a importância da bacia do rio São Francisco, que ocupa uma área aproximada de $638.576 \mathrm{~km}^{2}$, e afeta diretamente o desenvolvimento econômico de 521 municípios distribuídos em seis estados do Nordeste brasileiro, região severamente afetada pelo fenômeno da seca (AGÊNCIA NACIONAL DE ÁGUAS, 2017).

As duas bacias mencionadas têm em comum que percorrem grandes extensões, boa parte em terreno de baixa declividade, o que dificulta a extração computacional da rede de drenagem e delimitação de bacias por métodos convencionais.

A lei $n^{\circ}$ 9.433, de 8de janeiro de 1997, atribui à Bacia Hidrográfica (BH) o caráter de unidade territorial para implementação da política nacional e atuação do sistema nacional de gerenciamento de recursos hídricos, e, consequentemente, as atividades de pesquisa, monitoramento e alertas de processos hidrológicos (Jorge et al., 2015).

A maior frequência de eventos extremos de precipitação, potencialmente agravados pelas mudanças climáticas (TOMINAGA, SANTORO, AMARAL, 2009), levanta a questão sobre a necessidade de incorporar pesquisas relacionadas com redução do risco de desastre hidrológico no gerenciamento de bacias hidrográficas no Brasil. Em estudo realizado pelo Centro de Estudos e Pesquisas em Engenharia e Defesa Civil, da Universidade Federal de Santa Catarina - CEPED SC, junto ao Banco Mundial, foi constatado que entre 1995 e 2014 o Brasil teve um prejuízo aproximado de R\$182,8 
bilhões, relacionado a desastres naturais (CEPED UFSC, Relatório de Danos, 2016 (SCHADECK, 2016)). Além do valor econômico, é preocupante a quantidade de pessoas desabrigadas no Brasil por conta de enchentes ou de inundações graduais: 1,4 milhões somente entre 2008 e 2012 (IBGE, 2014).

Este trabalho teve como objetivo a geração de dados de delimitação de $\mathrm{BH}$, necessários à simulação de cenários de risco hidrológico, a partir de uma abordagem estocástica, contraposta à tradicional determinística. Com base na distribuição de probabilidade do conjunto de simulações, é possível identificar a frequência com que cada célula de terreno contribui à vazão no exutório da bacia. Nesse sentido, a simulação de diversos cenários de risco de inundação representa um recurso importante ao desenvolvimento de políticas públicas para redução do risco de desastres.

\section{Materiais e métodos}

Há diversas maneiras de promover a delimitação de bacias hidrográficas, desde o traçado manual, a partir de cartas topográficas, no qual os pontos de sela e os topos de morro são conectados, até a extração semiautomática, pela aplicação de algoritmos que avaliam modelos digitais de elevação (MDE) (Rosim et al.,2008).

O algoritmo “D8” (deterministic eight neighbours), de Jenson e Domingue (1998), é o mais utilizado na determinação da direção de escoamento a partir de MDE. Tal algoritmo avalia as oito células vizinhas a uma célula central e calcula qual a declividade em cada direção, atribuindo o fluxo à direção de maior declividade.

Dentre as mais conhecidas alternativas ao D8 estão os algoritmos baseados no MS-Quinn (Quinn et al., 1991), que possibilitam o traçado do escoamento multidirecional (MD8) por partição de fluxo em um mesmo evento de precipitação (Paz \& Collischonn, 2008).

Conforme panorama apresentado em Seiber t\& McGlynn (2007), ainda há na literatura diversas outras estratégias de determinação de fluxo. Entretanto, nesses trabalhos a BH ainda é tida como um objeto geográfico rígido, "binário", isto é, uma 
célula faz parte da bacia hidrografia ou não faz. Este determinismo por muitas vezes não condiz com a complexidade da natureza.

A abordagem estocástica proposta neste artigo é fundamentada na hipótese de que a direção de fluxo em cada célula pode ser dependente não apenas das diferenças de altimetria entre a célula e seus vizinhos. A forma escolhida para modelar a influência desses outros fatores, como, por exemplo, o evento de precipitação, foi a inclusão de uma componente estocástica à dinâmica (Ross, 2010). Desta forma, as direções de fluxo podem assumir diferentes valores a cada evento.

Para testar a abordagem estocástica de delimitação de BH foi construído um MDE hipotético. O MDE, conforme ilustrado na Figura 1, é representado por uma grade regular, na qual cada ponto tem associado um valor de elevação, em tons de cinza, tão mais escuros quanto menor a elevação da célula.

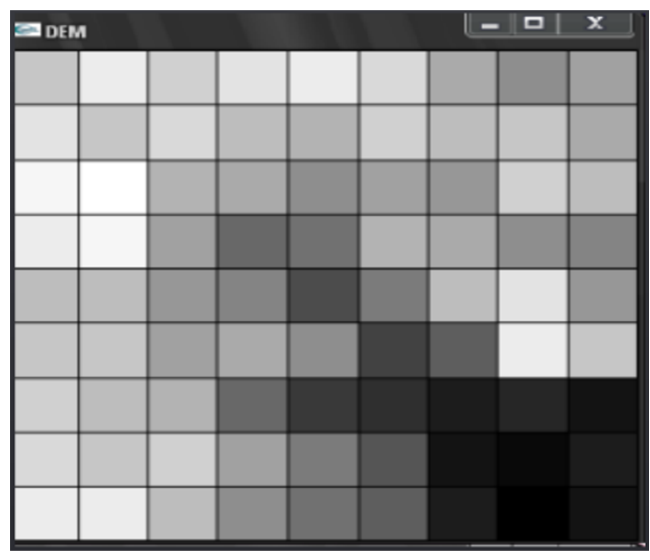

Fig. 1 - Ilustração em cores do Modelo Digital de Elevação (MDE) utilizado. A cada célula é associado um tom de cinza, do branco para valores mais altos até o preto para valores mais baixos.

Considerando um MDE conforme uma grade quadrada regular, de $\mathrm{N}$ por $\mathrm{N}$ células, cada célula do interior do terreno tem 8 vizinhos, correspondentes às 4 direções cardeais (norte, sul, leste e oeste) e às 4 colaterais (nordeste, noroeste, sudeste e sudoeste). A direção de fluxo é considerada única em cada evento, mas pode variar entre diferentes eventos. A probabilidade, $\mathrm{p}$, da direção de fluxo de uma célula $i, j$ apontar para uma célula $i+r, j+s$ pode ser, como dado na Equação 1, determinada em função da 
diferença de elevação entre a célula de origem $m d e(i, j)$ e célula de destino $m \operatorname{de}(i+$ $r, j+s)$, de forma a permitir o fluxo apenas de células de maior altimetria para células de menor altimetria:

$$
p_{i, j \rightarrow i+r, j+s}=\frac{\operatorname{máx}\left(0, M D E_{i, j}-M D E_{i+r, j+s}\right)}{\sum_{\{r, s\}=-1}^{1} \operatorname{máx}\left(0, M D E_{i, j}-M D E_{i+r, j+s}\right)}
$$

$$
\forall \mathrm{i}, \mathrm{j} \in \mathrm{N} \text { e } r, s \in\{-1,0,1\}
$$

Sujeito à condição complementar de que a água tem que escoar para algum vizinho, logo a soma da probabilidade de escoamento em cada célula, considerando todas as direções possíveis, tem que resultar na unidade, conforme a Equação 2:

$$
\sum_{\{r, s\}=-1}^{1} p_{i, j \rightarrow i+r, j+s}=1 ; \forall i, j \in N
$$

\subsection{Formulação estocástica}

A probabilidade definida anteriormente trata do Problema Direto (PD). Pode-se definir como PD quando, a partir de “causas" conhecidas, é possível calcular os "efeitos". Por exemplo: quando uma diferença de temperatura ("causa") é estabelecida em um material, o fluxo de calor ("efeito") será estabelecido do ponto mais quente ao ponto mais frio. Se, entretanto, o objetivo for descobrir, por exemplo, qual o valor da diferença de temperatura que gerou um fluxo de calor, então tal situação consiste no Problema Inverso (PI) associado (Campos Velho, 2008).

Para a solução do PIna configuração da $\mathrm{BH}$, é preciso determinar qual a probabilidade de uma célula do terreno contribuir hidrologicamente para o exutório (prédeterminado). Com base nos teoremas da probabilidade total e condicional, tem-se que 
tal probabilidade, em um dado ponto, é a soma das probabilidades de cada vizinho contribuir com o exutório dado que a água foi do ponto em questão ao vizinho em questão, conforme a Equação 3:

$$
q_{w v}=\sum_{\{k, l\}=-1}^{1}\left[\left(p_{w, v \rightarrow w+k, v+l}\right) \times q_{w+k, v+l}\right]
$$

$$
\forall w, v \in \mathrm{N} \text { e } \mathrm{k}, l \in\{-1,0,1\}
$$

Sujeito à condição complementar de que tal valor seja conhecido para uma das células do terreno, o exutório, e que $q_{w v}$ neste ponto seja igualà unidade, conforme a Equação 4:

$$
\exists w^{*} e v^{*} \in \mathrm{N} \mid q_{w^{*} v^{*}}=1
$$

Para análise da configuração mais frequente (configuração modal) da distribuição de configurações de bacias hidrográficas, ainda foi efetuada, conforme as Equações 1 e 2, uma simulação via roleta (Ross, 2010), na qual números aleatórios equiprováveis são gerados via código computacional e o escoamento é, então, simulado. O número de simulações efetuado foi de 100.000 processos, e o resultado foi armazenado na forma de frequência relativa.

\section{Resultados e discussões}

A unicidade da $\mathrm{BH}$ foi contestada pela abordagem estocástica proposta neste trabalho. Dado um terreno e um exutório no terreno, há diversas configurações possíveis da bacia à montante de tal exutório. Isto é, a BH já não é representada por um objeto geográfico fixo, do qual cada elemento do terreno tem uma opção binária de pertinência. 
A Figura 2 apresenta o mapa de probabilidade de pertinência de cada ponto do terreno à BH à do exutório (indicado pela letra “X”). A delineação em vermelho (mais externa) compreende a configuração da bacia determinística, enquanto que a delineação em verde (mais interna) representa a bacia modal. Nota-se que a célula destacada com a letra "a" faz parte da bacia determinística, ou seja, contribui com o escoamento até o exutório pela abordagem tradicional. Entretanto, na abordagem estocástica, tal célula não faz parte sequer da bacia mais frequente (modal). A célula destacada com a letra "b" participa tanto da bacia determinística quanto da modal, contribuindo com escoamento até o exutório em $57 \%$ dos casos (o mesmo percentual obtido para a célula destacada com a letra "a", que não faz parte da bacia modal). A célula destacada com a letra "c" não faz parte nem da bacia modal nem da bacia determinística, embora, na abordagem estocástica, contribua com o escoamento até o exutório em $45 \%$ dos casos (o mesmo percentual referente à célula “d”, que faz parte tanto da bacia modal quanto da determinística).

O valor mínimo encontrado nessa matriz de dados foi $0 \%$, ou seja, há células que nunca fazem parte da bacia hidrográfica em questão. O valor máximo foi 100\%, referente ao exutório - que, por definição, sempre faz parte da bacia - e a outras sete células contíguas a ele. O valor médio foi $48 \%$, com um desvio padrão de $42 \%$.

O fato da configuração determinística não ser igual à configuração modal mostra, na hipótese deste artigo, que a abordagem tradicional usa como bacia apenas uma configuração dentre um número muito grande de configurações possíveis, e tal configuração sequer é a mais frequente desse conjunto.

Além disso, diversas células (como no caso da célula destacada com a letra "c") apesar de não fazerem parte da bacia na abordagem tradicional, têm, na abordagem estocástica, uma frequência razoavelmente alta de contribuição com o escoamento até o exutório. 


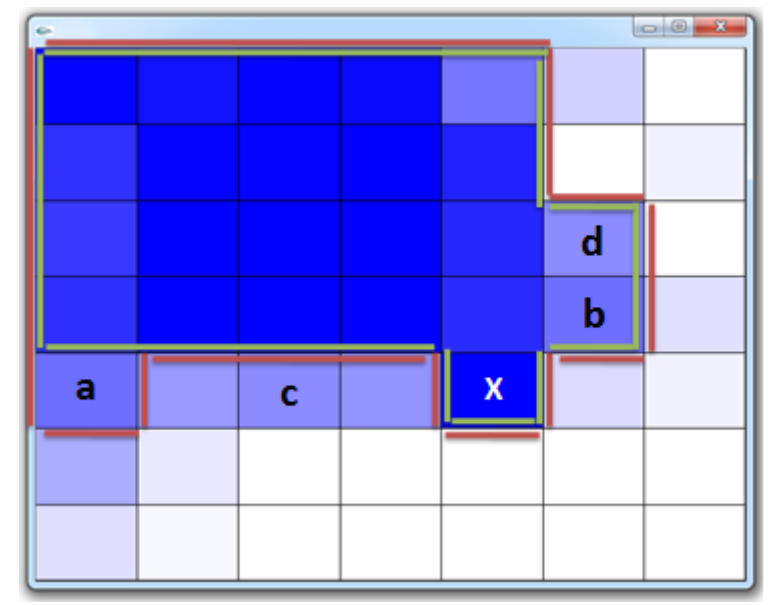

Fig. 2 - Ilustração em cores do mapa de probabilidade de pertinência de cada célula à bacia hidrográfica à montante da célula sinalizada com um " $X$ " branco (exutório). Quanto mais forte 0 tom de azul maior o valor da probabilidade. Em vermelho o contorno (mais externo) da bacia determinística (abordagem tradicional) e em verde o contorno (mais interno) da bacia modal.

\section{Conclusões e perspectivas}

O presente artigo propôs uma abordagem estocástica para o processo de delimitação de bacia hidrográfica, analisando tal abordagem tanto na perspectiva do problema direto quanto do problema inverso.

Em um contexto de maior frequência de eventos extremos de precipitação, potencialmente agravados pelas mudanças climáticas, a possibilidade de simular diferentes cenários de inundações motiva o desenvolvimento de novas ferramentas e, para esta finalidade, a abordagem estocástica proposta neste trabalho mostra-se uma ferramenta promissora.

O objeto geográfico bacia hidrográfica, tradicionalmente rígido, foi apresentado conforme um mapa de probabilidades. Foi evidenciada a diferença entre a configuração determinística (tradicional) e a configuração modal (mais frequente dentre todas as diferentes configurações das bacias).

A abordagem apresentada pode ser particularmente interessante no tratamento de terrenos planos, nos quais pequenas diferenças altimétricas podem proporcionar grandes diferenças em regimes de escoamento. Além disso, essa é uma abordagem que também pode ser utilizada em casos com incertezas nos valores de altimetria do modelo 
digital de elevação - a presença das incertezas demanda um tratamento probabilístico aos resultados de processamentos que têm tais dados como dados de entrada, como no caso da delimitação de bacias hidrográficas.

A consideração do mapa de probabilidades permite discutir a relevância da contribuição ao escoamento até o exutório de células que não pertencem à bacia hidrográfica tradicional. Tal abordagem pode ser utilizada, por exemplo, em modelos hidrológicos complexos compondo o mapa de precipitação com o mapa de probabilidades referentes à pertinência de cada célula à bacia hidrográfica em questão: essa é uma das perspectivas desta investigação.

\section{Agradecimentos}

Os autores agradecem o apoio do CNPq na forma de bolsas PIBIC aos alunos de graduação autores do artigo, e reconhecem, ainda, as valiosas contribuições dos revisores do manuscrito.

\section{Referências}

AGÊNCIA NACIONAL DE ÁGUAS. Bacia Hidrográfica Amazônica.2017. Disponível: http://www2.ana.gov.br/Paginas/portais/bacias/amazonica.aspx Acesso: Janeiro 2017

AGÊNCIA NACIONAL DE ÁGUAS. Bacia Hidrográfica do São Francisco.2017. Disponível:http://www2.ana.gov.br/Paginas/portais/bacias/SaoFrancisco.aspx.> Acesso: Janeiro 2017

CAMPOS-VELHO, H. F. Problemas inversos em pesquisa espacial. São Carlos: Sociedade Brasileira de Matemática Aplicada e Computacional, 2008. Disponível:

<http://www.lac.inpe.br/ haroldo/CursoPI/CursoPI.pdf>. Acesso: setembro 2015.

IBGE. Perfil de informações básicas unicipais 2013. Rio de Janeiro, 2014. Disponível: <http://biblioteca.ibge.gov.br/visualizacao/livros/liv86302.pdf>. Acesso: Janeiro 2017 
JENSON, S. K.; DOMINGUE, J. O. Extracting topographic structure from digital elevation data for geographic information system analysis. Photogramm. Eng. Remote Sens., v. 54, n. 11, p. 1593-1600, 1998.

JORGE, A. A. S.; CRUZ, A. C. S.; ANAYA, L. M.; RODRIGUES, J.; SANTOS, L. B. L. Classificação de Strahler para hidrografias brasileiras relacionadas a desastres naturais. Modelling in Science Education and Learning, v. 8, n. 1, 2015.

PAZ, A. R.; COLLISCHONN, W. Derivação de rede de drenagem a partir de dados do SRTM. Rev. Geogr. Acadêmica, v. 2, n. 2, p. 84-95, 2008.

QUINN, P.; BEVEN, K.; CHEVALLIER, P.; PLANCHON, O. The prediction of hillslope flow paths for distributed hydrological modelling using digital terrain models. Hydrological Processes, v.5, p. 59-79, 1991.

ROSIM, S.; MONTEIRO, A. M. V., RENNÓ, C. D., OLIVEIRA, J. R. F. Uma ferramenta open source que unifica representações de fluxo local para apoio à gestão de recursos hídricos no Brasil. Informática Pública, v. 10, p. 29-49, 2008.

ROSS, S. M. Introduction to probability models. 10th Ed. Burlington, MA, USA: Academic Press is an imprint of Elsevier. ISBN: 978-0-12-375686-2, 2010.

SANTOS, L. B. L.; MIRANDA, T. N. S.; FRATARI, J. C. D.; BACELAR, R. B.; MEDINA, C. B.; CARVALHO, S. V. HydroC - Biblioteca hidrológica aberta com fins educacionais. Modelling in Science Education and Learning, v. 8, n. 1, 2015.

SCHADECK, R. (Org.). Relatório de danos, materiais e prejuízos decorrentes de desastres naturais no Brasil 1995-2014. Florianópolis: CEPED UFSC, 2016. Disponível:

<http://www.ceped.ufsc.br/wp-content/uploads/2017/01/111703-WP-

CEPEDRelatoriosdeDanoslayout-PUBLIC-PORTUGUESE-ABSTRACT-SENT.pdf> Acesso: Janeiro 2016

SEIBERT, J.; MCGLYNN, B. L. A new triangular multiple flow direction algorithm for computing upslope areas from gridded digital elevation models.

WaterResourcesResearch. v.43, p. 1-8, 2007.

TOMINAGA, L. K.; SANTORO, J.; AMARAL, R. Desastres naturais: conhecer para prevenir. São Paulo: Instituto Geológico, 2009. ISBN: 978-8587235-09-1. 
Universidade do Estado de Santa Catarina - UDESC Centro de Ciências Humanas e da Educação - FAED

Revista PerCursos

Volume 18 - Número 36 - Ano 2017 revistapercursos@gmail.com 